BULL. AUSTRAL. MATH. SOC.

VOL. 25 (1982) 265-270.

\title{
A NOTE ON 1-FACTORS OF POINT DETERMINING GRAPHS
}

\section{Chia Gek-Ling and Lim Chong-Keang}

\begin{abstract}
A graph is called point determining if distinct points have distinct neighborhoods. In this note we provide a sufficient condition for a point determining graph to have a 1-factor. This is an extension of some results obtained by David P. Sumner.
\end{abstract}

All graphs considered in this note will be finite, undirected and without loops or multiple edges. Undefined terms can be found in [2]. If $G$ is a graph, then the neighborhood of $x$ in $G$ is denoted by $N(x)$. $G$ is called point determining if distinct points of $G$ have distinct neighborhoods. If $G$ is not point determining, we can obtain a point determining graph $\pi(G)$ from $G$ by identifying points $x$ and $y$ of $G$ with $N(x)=N(y)$. The set $\{a, b\}$ with $N(a)=N(b)$ is known as a $\pi$-pair of $G$. If $G$ is a point determining graph, we let $G^{0}=\{v \in G \mid G-v$ is point determining $\}$. In [4], Sumner gives some sufficient conditions on $G^{0}$ for a point determining graph $G$ to have a I-factor. His results are summarized in Theorem 1 below. In this note we shall extend his results by showing that if $G^{0}$ has no two disjoint $\pi$-pairs, and if $\pi\left(G^{0}\right)$ is a finite disjoint union of complete graphs of even order, then $G$ has a l-factor.

Throughout this note, $G+H$ will denote the join of $G$ and $H$ (see for example [2], p.21).

Received 20 October 1981. 
THEOREM 1 ([4], Theorems $3,4,5,7)$. Let $G$ be a point determining graph such that $G^{0}$ is any one of the following:

$$
\begin{aligned}
& \text { (i) } K_{2 n}+\bar{K}_{m}, 1 \leq m \leq 2, n \geq 0, \\
& \text { (ii) } K_{2} \cup K_{2}, \\
& \text { (iii) } K_{1,3},
\end{aligned}
$$

then $G$ has a 1-factor.

DEFINITION. Let $G$ be a point determining graph and $p \in G-G^{0}$. Then by ([4], Lemma 5), any two $\pi$-pairs in $G-p$ are disjoint. Let the $\pi$-pairs of $G-p$ be $\pi_{i}=\left\{p_{i}, q_{i}\right\}, i=1,2, \ldots, n$. Suppose further that these $\pi$-pairs are labelled in such a way that if exactly one element of $\pi_{i}$ is in $G^{0}$, then $q_{i}$ is that point. If both or neither of the elements of $\pi_{i}$ are in $G^{0}$, then let $p_{i}$ be the element of $\pi_{i}$ which is adjacent to $p$. We define $\pi(G ; p)=G-\left\{p, p_{1}, \ldots, p_{n}\right\}$.

LEMMA 1 ([4], Theorem 1 (b)). Let $G$ be a point determining graph and $p \in G-G^{0}$. Then $(\pi(G ; p))^{0} \subseteq G^{0}$.

LEMMA 2 ([4], Lemma 8). If $G$ is a point determining graph and $G^{0}$ has no two disjoint $\pi$-pairs, then for every $p \leqslant G^{0}, G-p$ has a unique $\pi$-pair.

LEMMA 3 ([3], Theorem 3). If $G$ is point determining then no point of $G-G^{0}$ is adjacent to every point of $G^{0}$.

THEOREM 2. Let $G$ be a point determining graph with $G^{0}=K_{2 n+1}+\bar{K}_{3}(n \geq 0)$. Then $G$ has a 1-factor.

Proof. By Theorem 1 ( $i i i)$, we may assume that $n>0$. The result is true for graphs of low order. Suppose that $G$ is a graph with $G^{0}=K_{2 n+1}+\bar{K}_{3}$ where $K_{3}=\{x, y, z\}$ and that the result holds for all graphs of order less than $|G|$. Since $G^{0}$ is not point determining, $G \neq G^{0}$ and let $p \in G-G^{0}$. Now Lemma 2 asserts that $G-p$ contains a 
unique $\pi$-pair, say $\{a, b\}$ with $N(a)=N(b)-p$.

We first observe that $K_{2 n+1}=G^{0}-\{x, y, z\} \subseteq(\pi(G ; p)\}^{0}$.

To see this, let $v \in K_{2 n+1}=G^{0}-\{x, y, z\}$. Then $v$ is adjacent to every other elements of $G^{0}$, so that if $v$ is an element of the $\pi$-pair in $G-p$, then the other element of the $\pi$-pair is not in $G^{0}$. Hence by the definition of $\pi(G ; p), v \in i(G ; p)$. By Lemma 1 , $(\pi(G ; p))^{0} \subseteq G^{0}$. Since no element of $\pi(G ; p)$ is adjacent to all of $(\pi(G ; p))^{0}$ by Lemma 3 , it follows that $v \in(\pi(G ; p))^{0}$.

We then have two cases to consider.

(I) $(\pi(G ; p))^{0}=G^{0}$.

In this case, the induction hypothesis asserts that $\pi(G ; p)$ has a 1-factor. This I-factor of $\pi(G ; p)$ together with the edge $b p$ forms a 1-factor of $G$.

(II) $(\pi(G ; p))^{0} \nsubseteq G^{0}$.

Recall that $(\pi(G ; p))^{0}$ contains $K_{2 n+1}=G^{0}-\{x, y, z\}$. Now if $(\pi(G ; p))^{0}=G^{0}-\{x, y, z\}=K_{2 n+1}$, then one of $x, y, z$ is a point of $\pi(G ; p)$ adjacent to all of $(\pi(G ; p))^{0}$, which is not possible by Lemma 3 . Hence $(\pi(G ; p))^{0}$ is either $K_{2 n+1}+\bar{K}_{1}$ or $\bar{K}_{2 n+1}+\bar{K}_{2}$. In any case, $\pi(G ; p)$ has a l-factor by Theorem 1 .

Now if $b \notin G^{0}$, then $\pi(G ; p) \simeq G-\{b, p\}$ and $b p$ together with a 1-factor of $\pi(G ; p)$ forms a l-factor of $G$.

Suppose $b \in G^{0}$. If $a \in G^{0}$, then again $\pi(G ; p) \simeq G-\{b, p\}$ and $G$ has a 1-factor. If $a \notin G^{0}$, then $\pi(G ; p) \simeq G-\{a, p\}$. Let $\left\{e_{1}, e_{2}, \ldots, e_{m}\right\}$ be a 1-factor of $\pi(G ; p)$ with $e_{1}=b w$ say. Then $\left\{a w, b p, e_{2}, \ldots, e_{m}\right\}$ is a 1-factor of $G$.

By induction, the theorem follows. 
THEOREM 3. Let $G$ be a point determining graph with $G^{0}$ containing no two disjoint $\pi$-pairs. Then $\pi\left(G^{0}\right)$ is a complete graph of even order if and only if $G^{0}=K_{2 n+1}+\bar{K}_{m}$ for some $n \geq 0$ and some $1 \leq m \leq 3$.

Proof. If $G^{0}=K_{2 n+1}+\bar{K}_{m}$, then clearly the result is true.

Conversely, suppose $\pi\left(G^{0}\right)$ is a complete graph of even order. If $G^{0}$ is point determining, then $\pi\left(G^{0}\right)=G^{0}=K_{2 n+1}+\bar{K}_{1}$. Hence assume that $G^{0}$ is not point determining. Let the $\pi$-pairs of $G^{0}$ be $\pi_{1}, \ldots, \pi_{t}$, $t \geq 1$. By the assumption on $G^{0}$, we have

(i) $\pi_{i} \cap \pi_{j} \neq \varnothing$,

(ii) $\pi_{i} \neq \pi_{j}$ for every $i \neq j$.

If $t=1$, then $G^{0}=K_{2 n+1}+\vec{K}_{2}$. This is true because $\pi\left(G^{0}\right)$ is a complete graph of even order.

If $t \neq 1$, then $t \geq 3$. To see this, suppose $t=2$. Then by (i) and $(i i)$ we may let $\pi_{1}=\{p, q\}$ and $\pi_{2}=\{q, r\}$ where $p \neq r$. Since $N(p)=N(q)=N(r)$ holds in $G^{0}$, it follows that $\{p, r\}$. is also a $\pi$-pair of $G^{0}$ different from $\pi_{1}$ and $\pi_{2}$. This contradicts $t=2$.

We further observe that the following is true:

(iii) $\pi_{i} \cap \pi_{j} \cap \pi_{k}=\emptyset$ for every $i<j<k$.

Suppose (iii) is false and let $\pi_{i} \cap \pi_{j} \cap \pi_{k}=\{a\}$ for some $i, j$ and $k$. Then we have

$$
\pi_{i}=\left\{a, p_{i}\right\}, \pi_{j}=\left\{a, p_{j}\right\} \text { and } \pi_{k}=\left\{a, p_{k}\right\}
$$

say, where $p_{i}, p_{j}$ and $p_{k}$ are all distinct. Then $\left\{p_{j}, p_{k}\right\}$ is also a $\pi$-pair of $G^{0}$ with $\pi_{i} \cap\left\{p_{j}, p_{k}\right\}=\varnothing$ contradicting (i).

In view of (i), (ii) and (iii), we see that the $\pi$-pairs of $G^{0}$ can 
be written as $\left\{q_{1}, q_{2}\right\},\left\{q_{2}, q_{3}\right\}, \ldots,\left\{q_{t}, q_{1}\right\}, q_{i}$ all distinct and $t \geq 3$. Now if $t \geq 4$, then $\left\{q_{1}, q_{3}\right\}$ and $\left\{q_{2}, q_{4}\right\}$ are also $\pi$-pairs of $G^{0}$ which are disjoint. But this is not possible by (i). Hence $t=3$. Since $\pi\left(G^{0}\right)$ is a complete graph of even order, it follows that $G^{0}=K_{2 n+1}+\vec{K}_{3}$.

REMARK $1 . \pi(G)$ is a complete graph of even (respectively odd) order if and only if $G=K_{2 n+1}+\bar{K}_{m}$ (respectively $K_{2 n}+\bar{K}_{m}$ ) for some $m \geq 1$ and some $n \geq 0$. However $G$ contains no two disjoint $\pi$-pairs only when $1 \leq m \leq 3$.

Theorems 1, 2 and 3 lead to the following result.

THEOREM 4. Let $G$ be a point determining graph with $G^{0}$ containing no two disjoint m-pairs. If $\pi\left(G^{0}\right)$ is a complete graph of even order, then $G$ has a 1-factor.

Now if $G^{0}$ has no two disjoint $\pi$-pairs and that if $\pi\left(G^{0}\right)$ is a finite disjoint union of complete graphs of even order, then at most one component of $G^{0}$ is of the form $K_{2 n+1}+\bar{K}_{m}$ with $n \geq 0$ and $2 \leq m \leq 3$.

COROLLARY. Let $G$ be a point determining graph with $G^{0}$ containing no two disjoint $\pi$-pairs. If $\pi\left(G^{0}\right)$ is a finite disjoint union of complete graphs of even order, then $G$ has a l-factor.

REMARK 2. Let $G$ be the path on seven points. Then $\pi\left(G^{0}\right)=2 K_{2}$ and $G$ has no 1 -factor. This example shows that the condition on $G^{0}$ having no two disjoint $\pi$-pairs cannot be omitted.

PROBLEM. Let $G$ be a point determining graph with $G^{0}$ having no two disjoint $\pi$-pairs. If $\pi\left(G^{0}\right)$ has a 1 -factor, does $G$ admit a I-factor? 


\section{References}

[1] Dennis P. Geoffroy, "On 1-factors of point determining graphs", Discrete Math. 24 (1978), 167-175.

[2] Frank Harary, Graph theory (Addison-Wesley, Reading, Menlo Park, London, 1969).

[3] David P. Sumner, "Point determination in graphs", Discrete Math. 5 (1973), 179-187.

[4] David P. Sumner, "l-factors of point determining graphs", J. Combin. Theory Ser. B 16 (1974), 35-41.

Department of Mathematics, University of Malaya, Kuala Lumpur 22-11, Malaysia. 